\title{
CONFORMIDADE DO ESPAÇO INTERNO LIVRE DE TRATORES AGRÍCOLAS E ITENS DE SEGURANÇA OBRIGATÓRIOS SEGUNDO AS NORMAS NBR/ISO 4252 E NR 12
}

\author{
Jairo Luís Zanon Peripolli', Airton dos Santos Alonço², Gessieli Possebom ${ }^{2}$ \\ ${ }^{1}$ Departamento de Engenharia Rural, Universidade Federal de Santa Maria, 97105-900, Santa Maria, Brasil. \\ ${ }^{2}$ Departamento de Engenharia Florestal, Universidade Federal de Santa Maria, 97105-900, Santa Maria, Brasil.
}

*E-mail: jairoperipolli15@hotmail.com

Recebido em: 17/11/2016.

Aceito em: 17/04/2017.

\section{RESUMO}

Os tratores são máquinas agrícolas que possibilitam o aumento da produção de alimento em maiores áreas. Entretanto, para haver aproveitamento dessa ferramenta, os tratores devem seguir especificações técnicas que garantam melhores condições ergonômicas ao operador a fim de reduzir riscos de acidentes e melhorar a eficiência do trabalho. Assim, o objetivo deste estudo foi verificar se tais máquinas se enquadram nas normas técnicas NBR/ISO 4252 e NR 12, que definem as medidas do espaço livre interno da cabine de tratores agrícolas e a presença de itens obrigatórios neles. Foram selecionados quatro tratores: Massey Ferguson ${ }^{\circledR} 4275$, Massey Ferguson ${ }^{\circledR}$ 6713R, John Deere ${ }^{\circledR}$ 6130J e John Deere ${ }^{8}$ 6165J. Em cada trator, foram realizadas medições do espaço interno livre da cabine conforme estabelecido pela norma técnica NBR/ISO 4252 e verificados os itens obrigatórios exigidos pela NR 12. Todas as medidas do espaço interno livre da cabine dos tratores estavam dentro dos parâmetros estabelecidos pela NBR/ISO 4252 e em conformidade com os itens obrigatórios estabelecidos pela norma NR 12.

Palavras-chave: Ergonomia; Máquinas Agrícolas; Segurança do Trabalho.

\section{Introdução}

Com a necessidade de cultivar grandes áreas para a produção de alimentos, tornou-se necessário desenvolver máquinas agrícolas para substituir progressivamente o trabalho manual pelo trabalho mecanizado [1]. O trator, desenvolvido no século XIX, proporcionou uma evolução significativa na produção a partir de sua introdução na agricultura, pecuária e silvicultura, sendo a principal fonte de potência utilizada pelo agricultor em sua propriedade [1-2]. Com sua evolução, os tratores tornaram-se máquinas que, além de maior potência e utilidade, devem fornecer conforto e segurança ao seu operador, pois, este, quando expostos a um ambiente de trabalho adequado, pode produzir mais e melhor [1].

Como qualquer operação em que máquinas são utilizadas, o trator e seu operador formam uma unidade produtiva integrada, chamada sistema homem-máquina. Quando a operação de tratores agrícolas não se constituir em um sistema eficiente, o operador é exposto a uma elevada carga física e mental, consequentemente, apresentando menor produtividade $\mathrm{e}$ qualidade do trabalho. Com isso, a ocorrência de erros e acidentes é elevada, assim como a probabilidade do desenvolvimento de doenças ocupacionais $[1,3]$.
Motivada por esse confronto entre homem-máquina, a ergonomia preocupa-se em estudar a adaptação do trabalho ao homem, levando em consideração as capacidades e limitações deste, do equipamento e do ambiente em vista do aumento da produtividade e melhoria de segurança, saúde, conforto, eficiência e satisfação na realização de suas atividades [4-6].

Nesse contexto, o estudo ergonômico se faz necessário, pois age sobre os fatores que determinam a eficiência desse sistema. Com base nos estudos de análise ergonômica do trabalho, definindo-se as interações existentes no ambiente laboral, é possível identificar problemas e, posteriormente, realizar melhorias a partir do desenvolvimento de máquinas, ferramentas e sistemas de produção adequados às características dos trabalhadores envolvidos [7].

Assim, é de grande importância que as condições no ambiente de trabalho dos operadores de tratores agrícolas atendam a alguns critérios de conforto e segurança.

Características do assento do operador, do espaço interno livre da cabine e dos comandos obrigatórios são alguns itens a serem controlados para tornar esse trabalho ergonomicamente correto e atender às normas reguladoras das atividades agrícolas. 
Segundo a NR 31 [8], cabe ao empregador realizar avaliações dos riscos para a segurança e saúde dos trabalhadores e, com base nos resultados, adotar medidas de prevenção e proteção que garantam, para todas as atividades, locais de trabalho, máquinas, equipamentos, ferramentas e processos produtivos seguros e em conformidade com as normas de segurança e saúde.

Desse modo, o objetivo deste trabalho foi verificar o cumprimento de alguns requisitos estabelecidos pelas normas NBR/ISO 4252 [9], que define medidas para o espaço interno livre da cabine de tratores, e pela NR 12 [10], que estabelece itens obrigatórios para tais ferramentas.

\section{Metodologia}

O estudo foi desenvolvido na região central do estado do Rio Grande do Sul. Para o levantamento dos dados, foram selecionados tratores de diferentes modelos, pertencentes a duas marcas. São eles: John Deere ${ }^{\circ}$, modelos: 6130J e 6165J e Massey Ferguson ${ }^{\circledR}$, modelos: 4275 e 6713R (Tabela 1). A escolha dessas marcas e desses modelos se deu única e exclusivamente em função da disponibilidade na época em que foi desenvolvido o trabalho.

Tabela 1. Principais especificações técnicas dos tratores agrícolas avaliados no estudo.

\begin{tabular}{lcccc}
\hline \multicolumn{1}{c}{ Especificações } & MF 6713R & MF 4275 & $\begin{array}{c}\text { JD } \\
\text { 6130J }\end{array}$ & JD 6165J \\
\hline $\begin{array}{l}\text { Potência do motor } \\
\text { na rotação nominal - } \\
\text { cv (kW) }\end{array}$ & $132(97)$ & $75(56)$ & $\begin{array}{c}130 \\
(95,6)\end{array}$ & $167(123)$ \\
\hline Marca do motor & AGCO & $\begin{array}{c}\text { Perkins } \\
1104 A- \\
44\end{array}$ & $\begin{array}{c}\text { John } \\
\text { deere } \\
6068 T\end{array}$ & $\begin{array}{c}\text { John } \\
\text { deere } \\
6068 \mathrm{H}\end{array}$ \\
\hline Número de cilindros & 4 & 4 & 6 & 6 \\
\hline Massa total (kg) & 7282 & $\begin{array}{c}3750 / 412 \\
5\end{array}$ & 7000 & 9400 \\
\hline $\begin{array}{l}\text { Comprimento total } \\
\text { (mm) }\end{array}$ & 4851 & $\begin{array}{c}3975 / 417 \\
0\end{array}$ & 4921 & 5420 \\
\hline Largura (mm) & 2266 & $\begin{array}{c}1445 / 216 \\
7\end{array}$ & 2439 & 2406 \\
\hline Altura máxima (mm) & 2926 & 2600 & 2741 & 2930 \\
\hline $\begin{array}{l}\text { Distância entre eixos } \\
\text { (mm) }\end{array}$ & 2626 & $\begin{array}{c}2135 / 237 \\
0\end{array}$ & 2650 & 2685 \\
\hline Ano de fabricação & 2014 & 2015 & 2013 & 2014 \\
\hline
\end{tabular}

A partir da seleção dos tratores agrícolas estudados, foram realizadas medições do espaço interno livre da cabine conforme especificações da norma técnica NBR/ISO 4252 [9], sendo, para isso, utilizada uma trena metálica de três metros. No total, foram avaliados dez pontos distintos nos tratores, os quais estão representadas na Figura 1, elencados de "a" à "j".

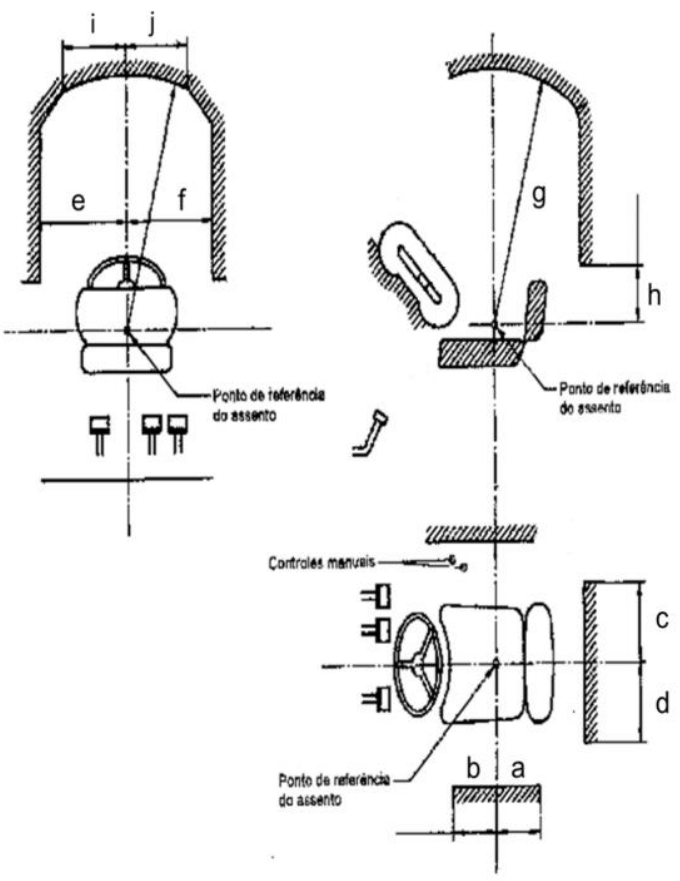

Figura 1: Identificação dos pontos de medição do espaço interno livre de tratores agrícolas, conforme estabelecido pela norma NBR/ISO 4252 [9]. *Ponto de referência do assento (SIP): ponto de referência determinado de acordo com a norma NBR/ISO 5353 [11].

Além disso, os tratores selecionados foram avaliados quanto à presença de itens obrigatórios estabelecidos pela NR 12 [10], que são: faróis, lanterna traseira de posição, buzina, espelho retrovisor, sinal sonoro de ré acoplado ao sistema de transmissão, estrutura de proteção na capotagem e proteção na parte superior e laterais da tomada de força. A partir dos dados coletados, foi realizada a comparação para aferir o cumprimento das normas.

\section{Resultados e discussões}

Embora as medidas estabelecidas para o espaço interno livre das cabines dos tratores agrícolas, instituídas pela NBR/ISO 4252 [9], não sejam baseadas em dados antropométricos de operadores da região de estudo, elas satisfazem as necessidades de espaço para esses operadores, pois, segundo [12], as medidas antropométricas dos operadores da região (Tabela 2) são muito próximas das medidas dos operadores americanos [13], utilizados como bases para a norma. 
Tabela 2: Medidas antropométricas de operadores de pulverizadores da região central do Rio Grande do Sul

\begin{tabular}{ccccccc}
\hline & \multicolumn{3}{c}{ Percentis } & & X & C.V. \\
\cline { 2 - 4 } Medida (cm) & $\mathbf{5 \%}$ & $\mathbf{5 0 \%}$ & $\mathbf{9 5 \%}$ & & & \% \\
\hline Altura do corpo & 168,0 & 176,0 & 191,0 & 177,0 & 7,5 & 4,2 \\
$\begin{array}{c}\text { Altura ao nível dos } \\
\text { olhos em pé }\end{array}$ & 160,3 & 168,5 & 180,0 & 169,3 & 7,2 & 4,2 \\
Altura ao nível dos & 77,0 & 88,0 & 97,0 & 87,9 & 6,3 & 7,2 \\
olhos sentado & 102,3 & 112,0 & 120,5 & 111,8 & 5,4 & 4,9 \\
Altura do cotovelo & 84,3 & 95,5 & 107,8 & 96,4 & 7,9 & 8,2 \\
Alcance do braço & 49,3 & 57,0 & 65,0 & 56,7 & 5,2 & 9,1 \\
Alcance da mão & 50,3 & 60,0 & 70,0 & 60,7 & 6,0 & 9,9 \\
Distância pé-rótula & 41,0 & 48,0 & 54,5 & 48,0 & 4,6 & 9,5 \\
Apoio do assento & 72,5 & 83,0 & 88,0 & 81,4 & 5,2 & 6,4 \\
Altura da coxa & 62,6 & 77,5 & 104,4 & 82,6 & 19,6 & 23,7 \\
\hline Massa corporal (kg) & & & & & \\
\hline
\end{tabular}

Em que: $\mathrm{X}=$ Média. $\mathrm{S}=$ Desvio Padrão. $\mathrm{CV}$ : Coeficiente de variação.

Conforme é possível observar na Tabela 3 , todas as medidas do espaço interno livre das cabines dos tratores seguiram as recomendações estabelecidas pela norma técnica NBR/ISO 4252 [9].

Tabela 3: Dimensões estabelecidas pela NBR/ISO 4252 [1] para o espaço interno livre da cabine de tratores agrícolas.

\begin{tabular}{|c|c|c|c|c|c|}
\hline $\begin{array}{c}\text { Itens } \\
\text { avaliados }\end{array}$ & $\begin{array}{c}\text { MF } \\
4275 \\
(\mathrm{~mm}) \\
\end{array}$ & $\begin{array}{c}\text { MF } \\
6713 R \\
(\mathrm{~mm}) \\
\end{array}$ & $\begin{array}{c}\text { JD } \\
6130 J \\
(\mathrm{~mm}) \\
\end{array}$ & $\begin{array}{c}\text { JD } \\
6165 J \\
(\mathrm{~mm}) \\
\end{array}$ & $\begin{array}{c}\text { NBR/ISO } \\
4252 \\
(\mathrm{~mm}) \\
\end{array}$ \\
\hline A & 180 & 140 & 140 & 140 & $\begin{array}{c}140 \\
\text { (mín.) }\end{array}$ \\
\hline B & 340 & 310 & 360 & 335 & $\begin{array}{c}310 \\
\text { (mín.) }\end{array}$ \\
\hline $\mathrm{C}$ & 423 & 485 & 430 & 430 & $\begin{array}{c}300 \\
\text { (mín.) }\end{array}$ \\
\hline D & 423 & 485 & 430 & 430 & $\begin{array}{c}300 \\
\text { (mín.) }\end{array}$ \\
\hline E & 738 & 450 & 710 & 710 & $\begin{array}{c}450 \\
\text { (mín.) }\end{array}$ \\
\hline $\mathrm{F}$ & 738 & 450 & 710 & 710 & $\begin{array}{c}450 \\
\text { (mín.) }\end{array}$ \\
\hline $\mathrm{G}$ & 1050 & 1050 & 1050 & 1050 & $\begin{array}{l}1050 \\
\text { (mín.) }\end{array}$ \\
\hline $\mathrm{H}$ & 300 & 300 & 300 & 300 & $\begin{array}{c}300 \\
\text { (máx.) }\end{array}$ \\
\hline I & 400 & 400 & 590 & 575 & $\begin{array}{c}330 \\
\text { (mín.) }\end{array}$ \\
\hline $\mathrm{J}$ & 400 & 400 & 590 & 575 & $\begin{array}{c}330 \\
\text { (mín.) }\end{array}$ \\
\hline
\end{tabular}

Em que: mín= valor mínimo exigido pela norma e máx= valor máximo exigido pela norma.
O posto de operação dos tratores agrícolas tem evoluído muito nos últimos anos, e a adequada distribuição e organização dos itens relacionados à ergonomia nos projetos contribuem para a melhoria do conforto e da segurança do operador [14]. O melhor projeto de cabines para máquinas e tratores agrícolas é, portanto, aquele em que o operador cometerá menos erros durante as tarefas de maior dificuldade, utilizando a mínima energia, e que cumpre o objetivo de organizar o local de trabalho em concordância com a natureza do operador e com a sua segurança.

É necessário, por parte dos fabricantes nacionais de tratores agrícolas, dar maior atenção à adequação dessas máquinas às exigências da Norma NBR 4252 [15]. O trator agrícola, estando em conformidade com a norma, não oferece apenas melhores condições ergonômicas aos operadores, evitando possíveis lesões corporais e acidentes laborais, como também proporciona melhor qualidade de trabalho aos operadores e estimula a satisfação pessoal, resultando no aumento de rendimento das atividades $[1,3,16]$.

Em relação aos itens exigidos pela NR 12 e avaliados neste estudo (faróis, lanterna traseira de posição, buzina, espelho retrovisor, sinal sonoro de ré acoplado ao sistema de transmissão, estrutura de proteção na capotagem e proteção na parte superior e laterais da tomada de força), todos estavam presentes nos diferentes modelos de tratores analisados.

Os acidentes e as lesões no meio rural são ainda pouco relatados, entretanto, conforme Couto [17], em torno de $20 \%$ do total de acidentes na agricultura são relativos a tarefas com tratores agrícolas, sendo 54\% classificados como leves. Dos acidentes graves, $14,8 \%$ são devido à condição de equipamentos inadequados ou ausência de itens de segurança obrigatórios. A manutenção adequada de todos os dispositivos que compõe uma máquina agrícola é de fundamental importância para a correta execução de sua função, o que, em conjunto, assegura a atividade do operador.

Segundo Cole; Myers; Westneat [18], o número de mortes se reduz em mais de $80 \%$ e as lesões fatais são cerca de $53 \%$ menores quando há a instalação da estrutura de proteção ao capotamento (EPC) em tratores mais antigos. No mesmo estudo, a EPC, juntamente com a cabina de segurança, mostrou-se eficiente na prevenção de lesões adicionais relacionadas à queda do operador do seu posto, destacando-se, dentre os dispositivos de segurança, como um dos mais importantes para a segurança do operador.

Percebe-se, nas pesquisas realizadas por [4, 19-22], que, quanto mais antigos os tratores agrícolas analisados, menor a incidência de dispositivos de segurança. Corroborando tais considerações, os tratores estudados apresentam ano de fabricação acima de 2013, fator possivelmente determinante para a total aferição da norma. 
Mesmo que tenham sido cumpridas as normas, são necessários novos estudos com um número maior de tratores, de diferentes marcas e modelos, além de se levarem em consideração outras normas técnicas envolvidas no estabelecimento de requisitos ergonômicos e de segurança a fim de assegurar ao operador que o maquinário disponibilizado no mercado siga os princípios da ergonomia e da segurança do trabalho.

\section{Conclusão}

Os tratores analisados possuem espaço livre interno das cabines condizentes com o exigido pela norma técnica NBR/ISO 4252, além de possuírem os itens obrigatórios instituídos pela NR 12. Dessa forma, são capazes de proporcionar aos operadores maior conforto e auxiliar na manutenção da saúde e bem-estar, diminuindo os riscos de acidentes de trabalho e aumentando o rendimento.

\section{Agradecimentos}

Proprietários dos tratores agrícolas estudados. Universidade Federal de Santa Maria - UFSM.

\section{CONFORMATION OF TO THE INTERNAL FREE SPACE OF AGRICULTURAL TRACTORS AND MANDATORY SECURITY ITEMS ACCORDING TO NBR/ISO 4252 AND NR 12 STANDARDS}

ABSTRACT: Tractors are agricultural machines that enable the increase of food production in larger areas. However, to be useful, tractors must follow technical specifications that ensure better ergonomic conditions to the operator in order to reduce the risk of accidents and improve work efficiency. Therefore, the objective of this study was to verify if such machines meet the technical standards NBR/ISO 4252 and NR 12, which define the measures of the internal space of the cab of agricultural tractors and the presence of items required. Four tractors ${ }^{1}$ were selected: Massey Ferguson ${ }^{\circledR}$ 4275, Massey Ferguson ${ }^{\circledR}$ 6713R, John Deere ${ }^{\circledR}$ 6130J and John Deere ${ }^{\circledR}$ 6165J. In each tractor, we measured the free internal space of the cab as established by the technical rule NBR/ISO 4252 and verified the mandatory items required by NR 12. All measurements of the internal free space of the tractor cab were in accordance with the parameters established by NBR/ISO 4252 and the mandatory items established by NR 12 .

Keywords: Ergonomics; Agricultural machinery; Occupational safety and health.

\section{Referências}

[1] ROZIN, D. Conformidade do posto de operação de tratores agrícolas nacionais com normas de ergonomia e segurança. 2004. 211 p. Dissertação de Mestrado em Engenharia Agrícola pela Universidade Federal de Santa Maria, Santa Maria. 2004.

[2] COSTA NETO, W. V. da. Estudo ergonômico na linha de montagem de tratores agrícolas. 2008. 124 f. Dissertação de Mestrado em Engenharia Agrícola pela Universidade Federal de Santa Maria, Santa Maria, 2008.

[3] DEBIASI, H.; SCHLOSSER, J. F.; PINHEIRO, E. D. Características ergonômicas dos tratores agrícolas utilizados na região central do Rio Grande do Sul. Ciência Rural, Santa Maria, v.34, n.6, p.1807-1811, 2004

[4] IIDA, I. Ergonomia: Projeto e produção. São Paulo: Edgard Blücher, 465p. 2016

[5] MARQUES, M. Abordagem ergonômica para a melhoria contínua das condições de trabalho em sistemas de gestão de qualidade. 2002. 76 p. Dissertação de Mestrado em Engenharia de Produção pela Universidade Federal do Rio Grande do Sul, Porto Alegre. 2002.

[6] SANTANA, A. M. C. A produtividade em unidades de alimentação e nutrição: Aplicabilidade de um sistema de medida e melhoria da produtividade integrando a ergonomia. 2002. 255 f. Tese de Doutorado em Engenharia de Produção pela Universidade Federal de Santa Catarina, Florianópolis, 2002.

[7] ZVIRTES, L. Sistemática para apoiar a identificação e a qualidade dos custos associados às ações ergonômicas. 2002. 105 f. Dissertação de Mestrado em Engenharia de Produção pela Universidade Federal do Rio Grande do Sul, Porto Alegre. 2002.

[8] BRASIL. Ministério do Trabalho e emprego. NR 31 - Segurança e saúde no trabalho na agricultura, pecuária, silvicultura, exploração florestal e aquicultura. Brasília: Ministério do Trabalho e Emprego, 2011. Disponível em:<http://trabalho.gov.br/seguranca-e-saude-no-trabalho/normatizacao/normasregulamentadoras >. Acesso em: 29 out. 2016.

[9] ABNT - ASSOCIAÇÃO BRASILEIRA DE NORMAS TÉCNICAS. NBR/ISO 4252: Tratores Agrícolas - Local de trabalho do operador, acesso e saída - Dimensões. Rio de Janeiro, 2011. 10 p.

[10] BRASIL. Ministério do Trabalho e Emprego. NR 12 - Segurança no trabalho em máquinas e equipamentos. Brasília: Ministério do Trabalho e Emprego, 2015. Disponível em: <http://trabalho.gov.br/seguranca-e-saude-no trabalho/normatizacao/normas-regulamentadoras/norma-regulamentadora-n-12seguranca-no-trabalho-em-maquinas-e-equipamentos>. Acesso em: 17 out. 2016.

[11] ABNT - ASSOCIAÇÃO BRASILEIRA DE NORMAS TÉCNICAS NBR/NM/ISO 5353: Máquinas rodoviárias, tratores e máquinas agrícolas e florestais - Ponto de referência do assento. Rio de Janeiro, 5 p. 1999.

[12] BAUMHARDT, U. B. Metodologia para concepção de cabines de máquinas agrícolas com enfoque na segurança e ergonomia. 2012. 259 p. Tese de Doutorado em Engenharia Agrícola pela Universidade Federal de Santa Maria, Santa Maria. 2012

[13] THOMAS, R. E. et al. The anthropometry of forest machine operators in the southern USA. Journal of Forest Engineering, v.5, p.33-41, 1994.

[14] MADEIRA, N.G. Segurança no trabalho nas operações com tratores agrícolas em regiões de Minas Gerais. Tese de Doutorado em Engenharia Agrícola pela Universidade Federal de Viçosa, Viçosa, 2011.

[15] MATTAR, Danielle M. P. et al. Conformidade de acessos e de saídas de postos de operação em tratores agrícolas segundo norma NBR/ISO 4252. Engenharia Agrícola., Jaboticabal, v.30, n.1, p.74-81, 2010.

[16] SILVA, C. B. et al. Avaliação ergonômica de uma colhedora de cana-deaçúcar. Ciência e Agrotecnologia, Lavras, v. 35, n. 1, p. 179-185, 2011. 
[17] COUTO, J.L.V. Riscos de acidentes com tratores agrícolas. Rio de Janeiro: Universidade Federal do Rio de Janeiro. Disponível em: $\langle$ http://www.segurancaetrabalho.com.br/download/tratorescouto.doc $>$ Acesso em: 10 out. 2016

[18] COLE. H. P.; MYERS. M. L. WESTNEAT. S. C. Frequency and severity of injuries to operators during overturns of farm tractors. Journal Health Saf Agric. 11p. 2006

[19] DEBIASI, H. Diagnóstico dos acidentes de trabalho e das condições de segurança na operação de conjuntos tratorizados. 2002. 284p. Dissertação de Mestrado em Engenharia Agrícola pela Universidade Federal de Santa Maria.

[20] LIMA, J. S. S., et al. Avaliação de alguns fatores ergonômicos nos tratores "Feller-Buncher" e "Skidder" utilizados na colheita de madeira. Revista Árvore, Viçosa, v.29, n.2, p.291-298, 2005.

[21] BRITO, A. B. Avaliação e redesenho da cabine do "Feller buncher" com base em fatores ergonômicos. 2007. 151p. Tese de Doutorado em Engenharia Mecânica pela Universidade Federal de Viçosa, Viçosa, MG.

[22] MONTEIRO et al., L. A. Prevenção de Acidentes com Tratores Agrícolas e Florestais. UNESP - BOTUCATU - São Paulo, 105p., 2010. 